\title{
A HAWAll-2RG infrared camera operated under fast readout mode for solar polarimetry
}

\author{
Yoichiro Hanaoka ${ }^{1 *} \mathbb{0}$, Yukio Katsukawa', Satoshi Morita', Yukiko Kamata' and Noriyoshi Ishizuka ${ }^{1,2}$
}

\begin{abstract}
Polarimetry is a crucial method to investigate solar magnetic fields. From the viewpoint of space weather, the magnetic field in solar filaments, which occasionally erupt and develop into interplanetary flux ropes, is of particular interest. To measure the magnetic field in filaments, high-performance polarimetry in the near-infrared wavelengths employing a high-speed, large-format detector is required; however, so far, this has been difficult to be realized. Thus, the development of a new infrared camera for advanced solar polarimetry has been started, employing a HAWAll-2RG $(\mathrm{H} 2 \mathrm{RG})$ array by Teledyne, which has $2048 \times 2048$ pixels, focusing on the wavelengths in the range of $1.0-1.6 \mu \mathrm{m}$. We solved the problem of the difficult operation of the H2RGs under "fast readout mode" synchronizing with highspeed polarization modulation by introducing a "MACIE" (Markury ASIC Control and Interface Electronics) interface card and new assembly codes provided by Markury Scientific. This enables polarization measurements with high frame-rates, such as 29-117 frames per seconds, using a H2RG. We conducted experimental observations of the Sun and confirmed the high polarimetric performance of the camera.
\end{abstract}

Keywords: Solar observation, Polarimetry, Magnetic field, Space weather, Near-infrared, H2RG

\section{Introduction}

Solar polarimetry is indispensable to determine solar magnetic fields governing a variety of phenomena in the solar atmosphere. Eruptive events occurring on the solar surface occasionally result in a harmful effect to the Earth, and thus, the investigation of such phenomena and the magnetic field information on the Sun is crucially important. From the viewpoint of space weather, to obtain accurate magnetic field information, the realization of advanced solar polarimetry, employing a highspeed, large-format detector covering near-infrared wavelengths is required.

Near-infrared polarimetry provides a new insight into the solar magnetic field study. Therefore, many attempts have been performed particularly in the past two decades with such instruments as the Tenerife Infrared Polarimeter (TIP; Mártinez Pillet et al. 1999) and TIP II (Collados

\footnotetext{
*Correspondence: yoichiro.hanaoka@nao.ac.jp

${ }^{1}$ National Astronomical Observatory of Japan, 2-21-1 Osawa, Mitaka, Japan

Full list of author information is available at the end of the article
}

et al. 2007) at the German Vacuum Tower Telescope of the Observatorio del Teide, the GREGOR Infrared Spectrograph (Collados et al. 2012) at the GREGOR telescope also of the Observatorio del Teide, and the Facility Infrared Spectropolarimeter (Jaeggli et al. 2010) installed at the Dunn Solar Telescope at Sacramento Peak.

One of the reasons of the advantage of the near-infrared observations is that there are a number of rather informative absorption lines in the near-infrared range. One of these is the He I 1083.0-nm line, which is formed in the chromosphere (e.g., Lagg 2007; Penn 2014). In addition to the information on the chromosphere, the He I 1083.0-nm line provides information on the magnetic field in solar filaments (e.g., Hanaoka and Sakurai 2017). Filaments occasionally erupt and become part of coronal mass ejections (CMEs), and the magnetic field in the filaments is the source of the magnetic field in the CME interplanetary flux ropes. From the viewpoint of space weather, the information on the magnetic field in the flux ropes arriving at the Earth is crucially important, because the orientation of the magnetic field in the flux ropes affects the severity of the resulting geomagnetic 
storms. To determine the magnetic field orientation in the interplanetary flux ropes in advance, the information on the magnetic field in the filaments before their eruption is an important factor. So far, pieces of indirect information, such as fine structures in filaments seen in $\mathrm{H} \alpha$, have been used to estimate the magnetic field in filaments; however, the direct measurements of the magnetic field in filaments using the He I 1083.0-nm line provide essential contributions to the prediction of the magnetic field of interplanetary flux ropes.

Another advantage of the He I 1083.0-nm line is that a close photospheric line, Si I $1082.7 \mathrm{~nm}$, can be detected simultaneously with it in spectroscopic observations. Observations using these two lines provide both photospheric and chromospheric magnetic field information. In addition, the Fe I 1564.8-nm line is also an important target in near-infrared wavelengths (see Harvey and Hall 1975 and also Penn 2014). Although it is a photospheric line, it shows a particularly large Zeeman splitting, which provides us a different method to investigate the magnetic field from other lines.

Besides the selection of target absorption lines, the performance of detectors is also important to realize advanced solar polarimetry. High speed is one of the key specifications required for detectors, because it contributes to the reduction of noise level. The polarization of the solar light is generally weak; thus, the typical required noise level is as low as $10^{-3}-10^{-4}$ (see e.g., Lagg et al. 2017). This results in the requirement of rapid polarization modulation. The polarization modulation produces a variation in the light intensity reaching the detector depending on the polarization state of the incident light. In solar polarimetry with ground-based telescopes, the image distortion due to the seeing effect during the polarization modulation produces unwanted variation in the light intensity. To reduce this seeing-induced error, fast polarization modulation and high frame rate are required. Their combination enables complete image acquisition before the image distortion becomes substantially large. The noise level $10^{-3}-10^{-4}$ indicates that photoelectrons at least in the range of $10^{6}-10^{8} \mathrm{e}^{-}$need to be accumulated. To realize this, many images need to be integrated; however, due to the extremely high brightness of the Sun, this can be performed within a short time with a high frame rate. The shortness of the data acquisition time enables a high-cadence observation, which is necessary to acquire data before the structure of the observing targets changes substantially.

In addition, large-format detectors are required to realize efficient data acquisition. Small-format cameras have already been successfully used for solar polarimetry. For example, the solar group of the National Astronomical Observatory of Japan (NAOJ) has been operating regular full-disk, full-Stokes polarimetry observations of the Sun at wavelengths including He I $1083.0 \mathrm{~nm}$ and Fe I $1564.8 \mathrm{~nm}$ with XEVA cameras by Xenics, equipped with a $640 \times 480$-pixel InGaAs detector (Sakurai et al. 2018). The instruments used at the Observatorio del Teide and Sacramento Peak mentioned above also use detectors with up to $1024 \times 1024$ pixels. However, the efficiency of observations with small-format cameras is not sufficiently high to track the evolution of the magnetic field.

Therefore, for solar polarimetry, a high-speed, largeformat infrared detector is required. The HAWAII-xRG focal plane arrays by Teledyne Imaging Sensors are largeformat, low-noise $\mathrm{HgCdTe}$ detectors for near-infrared wavelengths, and they can be operated with high frame rates. Among them, the HAWAII-2RG (H2RG) detector (Blank et al. 2012) has been used most commonly in astronomical observations. A H2RG has $2048 \times 2048$ pixels with a pixel size of $18 \times 18 \mu \mathrm{m}$, and its full-well capacity is $\sim 100,000 \mathrm{e}^{-}$. In night-time astronomical observations, it is used in the slow readout mode (readout speed of $100 \mathrm{kHz}$ ) where the readout noise is as low as $\sim 15 \mathrm{e}^{-}$. Nevertheless, a fast readout mode $(5 \mathrm{MHz})$ is also available, and with this mode, the frame rate can be as high as 33 frames per second (fps), with 32-channel simultaneous readouts. In the fast readout mode, the noise level increases to a certain extent $\left(\sim 70 \mathrm{e}^{-}\right)$and the depth of analog-to-digital (A/D) conversion is lowered to 12 bits from 16 bits in the slow readout mode. Nevertheless, this high frame rate is suitable to observe photon-rich targets such as the Sun. Therefore, the H2RG is expected to meet the requirements of a high-speed, large-format infrared detector for solar polarimetry.

However, it has been difficult to employ a H2RG for solar polarimetry. For the efficient and precise solar polarimetry, many images should be taken continuously synchronizing with the polarization modulation. However, a H2RG and its peripheral electronics were not necessarily designed for such an operation. Even in the fast readout mode, they are basically optimized for a single image acquisition, and it is difficult to carry out stable, uninterrupted continuous image acquisitions. Furthermore, there is no function to synchronize the exposure timing and an external device like a polarization modulator very precisely. On the other hand, some small-format cameras have a function to be synchronized with external signals under the stable continuous image-acquisition mode, and it is the reason why they have been used for solar polarimetry.

Our aim was to develop a mechanism for the synchronization, to realize a large-format, low-noise infrared camera for advanced solar polarimetry using a H2RG detector. To realize the synchronization in the fast readout mode of the H2RG, in cooperation with Kyoto 
University, we introduced a "MACIE" (Markury ASIC Control and Interface Electronics) card (Loose et al. 2018) manufactured by Markury Scientific. Finally, we realized the synchronization of the polarization modulation and image acquisition with a H2RG under the fast readout mode, as briefly reported by Hanaoka et al. (2019). We adopted a H2RG device with the cut-off wavelength of $1.7 \mu \mathrm{m}$, because we focused on the wavelengths in the range of $1.0-1.6 \mu \mathrm{m}$. In principle, our development had an experimental purpose; therefore, we chose an engineering model of H2RG (serial number \#17087). Nevertheless, such a development can realize efficient polarimetry with future advanced telescopes.

We present an overview of the camera system and an example of experimental polarimetric observations of the Sun in the following two sections and summarize our results in the conclusion section.

\section{Overview of the system and the principle of the synchronous operation}

The configuration of the camera system is shown in Fig. 1. We adopted a rotating waveplate driven by a stepper motor for the polarization modulator. The incident light goes through the rotating waveplate and an "analyzer" (a linear polarizer) and reaches the H2RG detector. As described before, in polarimetry the synchronization between the polarization modulation and image acquisition is crucially important.

We employed a MACIE card for the interface between a "SIDECAR" (System for Image Digitization, Enhancement, Control And Retrieval; Chen et al. 2014) application-specific integrated circuit (ASIC) focal plane electronics (which outputs A/D-converted signals) and a personal computer (PC). Typically a "SAM" (SIDECAR

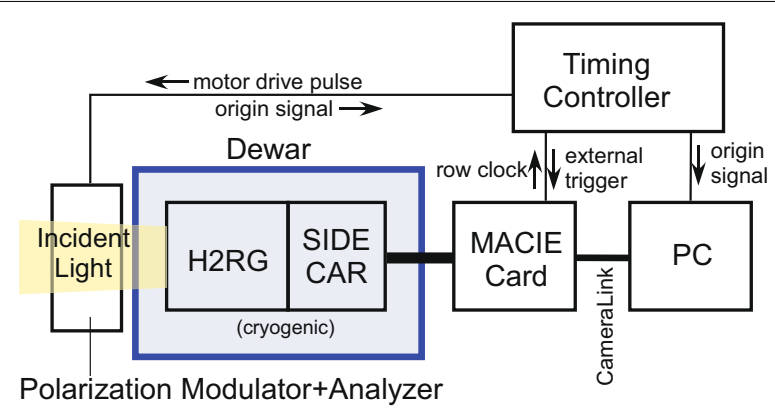

Fig. 1 Diagram of the camera system. The incident light travels through a polarization modulator and an analyzer. It is detected by a $\mathrm{H} 2 \mathrm{RG}$, and analog-to-digital conversion is carried out by a SIDECAR. They are installed in a cryogenic dewar. The digitized data are transferred from the SIDECAR to a PC relayed by a MACIE card via CameraLink. A timing controller receives the row clock from the MACIE card and controls the synchronous actions among polarization modulation, image acquisition, and data transfer
Acquisition Module) card by Teledyne (Blank et al. 2012) is used for the interface; however, it is difficult to synchronize the polarization modulation and image acquisition using a SAM card. Nevertheless, the MACIE card has a function to send and receive timing signals. The synchronization with the polarization modulator becomes possible using these signals. New assembly codes (firmwares) developed by Markury Scientific have been installed into the SIDECAR to realize the synchronizing operation.

The synchronization is managed by a timing controller. The timing controller functions are implemented by a Complex Programmable Logic Device (CPLD), which receives the "row clock" (line sync signal) from the MACIE card and provides motor drive pulses to the polarization modulator. It also produces trigger signals to start the image readouts synchronizing with the rotation of the waveplate and sends the signals to the MACIE card.

Figure 2 shows the timing relations among various signals and data readouts in detail. Using the 32-channel readout in fast readout mode, a H2RG and a SIDECAR run under the row clock of $\sim 70 \mathrm{kHz}$. One cycle of the row clock corresponds to the processing time of each row of the detector. Specifically, a H2RG/SIDECAR processes approximately 70,000 rows in a second in fast readout mode. The row clock is an internal signal produced by the SIDECAR; therefore, it can be used as the base clock to control the overall timing of the system.

When the MACIE card receives an external trigger signal, it starts the data readout of a frame by the SIDECAR. To drain out the photoelectrons, a reset of the detector is implemented at the row clock after the subsequent one of the readout at each row. An exposure starts after the reset signal and ends at the readout. The exposure needs to be longer than the time needed to read the data of all the rows, i.e., 2048 row clocks (and a margin) for a full frame. As the dead time between a "read" and a "reset" is only two row clocks, it is virtually negligible (the duty ratio, the ratio of the exposure time to the exposure interval, is $>99 \%$ ). Hence, this is a rather effective system, where most of the photons coming into the detector are used to produce images.

Nevertheless, such a "reset-read" operation has some shortcomings. The noise level in the "reset-read" operation is generally higher than that in the "reset-readread" operation (correlated double sampling, CDS), and therefore, the CDS is commonly used in night-time astronomical observations. In fast readout mode, the CDS also lowers the noise level to a certain extent; however, it is not very effective (Blank et al. 2012). Nevertheless, the CDS lowers the frame rate and increases the dead time. Another shortcoming is the residual image or persistency due to the residual photoelectrons, which 


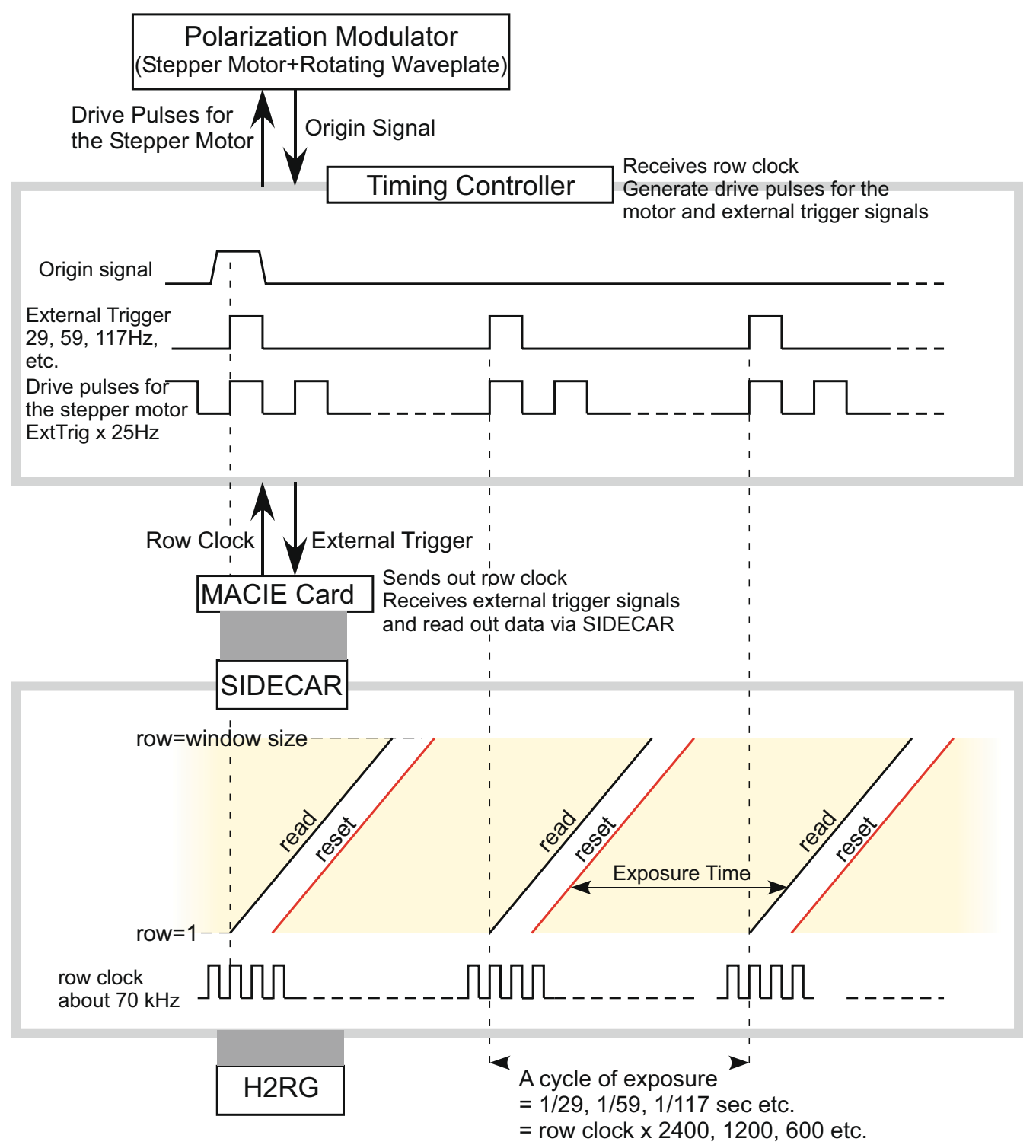

Fig. 2 Timing chart of the synchronous system including the timing controller, the polarization modulator, and the SIDECAR/MACIE with the H2RG

remain after the reset operation (e.g., Mosby et al. 2016), and which are also among the sources of noise on images. It is known that a single reset operation cannot completely drain out the photoelectrons accumulated during the exposure. Repeated reset operations are effective to reduce the residual electrons, and the CDS is also useful. However, these operations result in a reduction of the frame rate. Therefore, there is a trade-off relation between the noise and the frame rate. The effectiveness of the introduction of the CDS and/or multiple resets will be analyzed in future studies.

The polarization can be fully expressed by four Stokes parameters $(I, Q, U$, and $V)$, and to determine them, typically 16 images are taken during one rotation of the waveplate of a polarization modulator. In our case, one rotation of the waveplate is completed by 400 drive pulses for the stepper motor, and thus, the frame time to take an image corresponds to 25 drive pulses. In the case of a full-frame image (2048 rows), we typically set the frame time to be 2400 row clocks including a margin. It means that the exposure time starting at a "reset" and ending at a "read" (see Fig. 2) is 2398 (=2400 - 2 clocks of dead time) row clocks. The timing controller divides 2400 row clocks to 25 drive pulses in this case.

The rotating waveplate unit is equipped with an origin sensor. The waveplate hits the origin sensor once in 
every rotation, and the timing controller receives the origin signal. Then, the timing controller starts to send out the external trigger signals to start the image readout by the H2RG/SIDECAR every 25 drive pulses. The actual number of drive pulses between two origin signals, which needs to be 400 , is constantly monitored to detect any step-outs of the motor. In this manner, the external trigger signals are issued at the same phase angles in every rotation, and thus realizing the synchronization. The data acquired by the MACIE card are transferred to the PC. The $\mathrm{PC}$ receives the origin signal via the timing controller, and uses it as the trigger to start to record the transferred data.

As mentioned above, we typically set the number of clocks to be 2400 for a full-frame image. The number of row clocks of 2400 per frame corresponds to a frame rate of $29 \mathrm{fps}$ and a waveplate rotation of $1.8 \mathrm{rps}$. The new assembly codes for the SIDECAR enable vertical windowing. By decreasing the width of the window from 2048, the frame rate increases. For instance, a 1024-row window (1200 row clocks per frame) enables 59 fps with a rotation of $3.7 \mathrm{rps}$, and a 512-row window (600 row clocks per frame) enables $117 \mathrm{fps}$ with 7.3 rps.

The drive pulse frequency for all rotation speeds mentioned above is higher than the maximum pull-in pulse rate of the stepper motor. Therefore, the motor starts to rotate with low-frequency pulses produced by the timing controller, and then it is accelerated to a target speed such as $1.8 \mathrm{rps}$ for the full-frame readout. After reaching the target speed, the drive clock is gradually switched to that produced from the row clock. Thus, the synchronization between the waveplate rotation and the row clock is established. In this way, using the H2RG, we realized polarimetry with high frame rates such as $29-117 \mathrm{fps}$.

As shown in Fig. 2, the exposure of each row starts progressively. Such an operation corresponds to the rolling shutter, and each row undergoes different polarization modulations. To demonstrate the synchronization and the progressive readout, Fig. 3 shows measurement results of completely polarized artificial light. The incident polarized light (linear polarizations of Stokes $Q / I=1$ and $U / I=1)$ traveled through the rotating waveplate and the analyzer, and the light intensity reaching the detector varied sinusoidally due to the polarization modulation. We set the window of 512 rows $(2048 \times 512$ pixels), and the waveplate completed one rotation with 9600 row clocks $(600$ row clocks $\times 16$ images). The $x$-axis in Fig. 3 represents one rotation of the waveplate. At row clock 1 , row 1 of the first image was read, and at row clock 512, row 512 of the first image was read. At row clocks 601-1112, the second image was read and at row clocks 9001-9512, the last (16th) image was read. In Fig. 3, the measured light intensities averaged

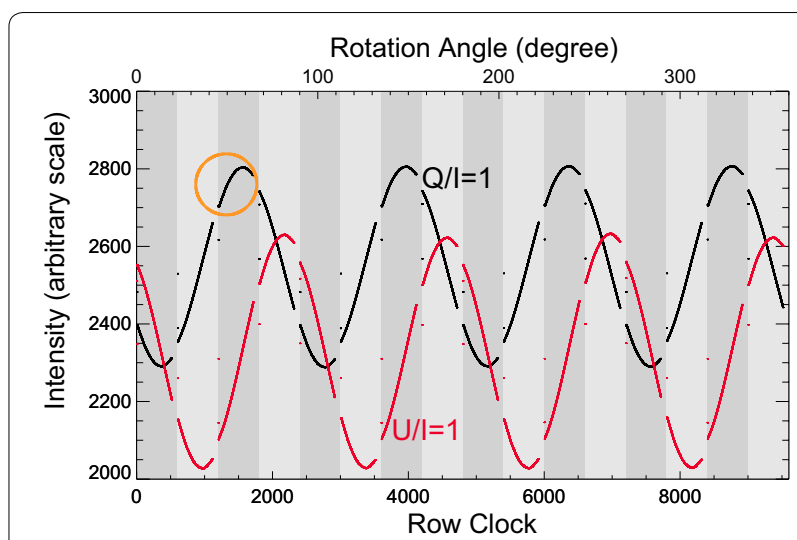

Fig. 3 Measurement results of linearly polarized artificial light (Stokes $Q / I=1$ and $U / I=1$ ). The range of the $x$-axis corresponds to one rotation of the waveplate, which includes 16 image acquisitions represented by vertical stripes. Each image has 512 rows, and the average light intensities detected by each row are plotted at the row clock corresponding to their readout timings. Each segment of the curves (one of them is marked by an orange circle) is comprised of 511 data points (row 1 contains dummy data). The measurement results for both the Stokes $Q / /$ and $U /$ I inputs show sinusoidal variations. Each line is similar to a single sinusoidal curve; however, the measurement results of 16 contiguous rotations are overplotted

every row are plotted at the row clock corresponding to their readout timings. Each segment of the curves (one of which is marked by an orange circle in Fig. 3) is comprised of 511 data points (row 1 contains dummy data) in an image, and as a whole, the measurement results for both Stokes inputs $Q / I$ and $U / I$ show sinusoidal variations as expected. In Fig. 3, the measurement results of 16 continuous rotations are overplotted. All data points of 16 rotations look like to fall on a single curve. The fluctuation of the measured signal during the 16 rotations is about $4 \times 10^{-4}$, even including the possible brightness fluctuation of the light source. This result indicates that the quality of the synchronization of the data readout and the polarization modulation is very high.

\section{Experimental observation and the verification of the performance}

To verify the performance of the camera system, experiments for the polarization measurement of the Sun were carried out using the Domeless Solar Telescope (DST; Nakai and Hattori 1984) of the Hida Observatory, Kyoto University in November 2018 and in November 2019.

The camera system and its experimental arrangement are shown in Fig. 4. The H2RG and the SIDECAR were installed in a dewar (manufactured by OptCraft). A cryocooler (a helium Stirling cooler by Twinbird) cooled the $\mathrm{H} 2 \mathrm{RG}$ down to $-130^{\circ} \mathrm{C}$ to reduce thermal noise. As the frame rate is high and the cut-off wavelength is as short 


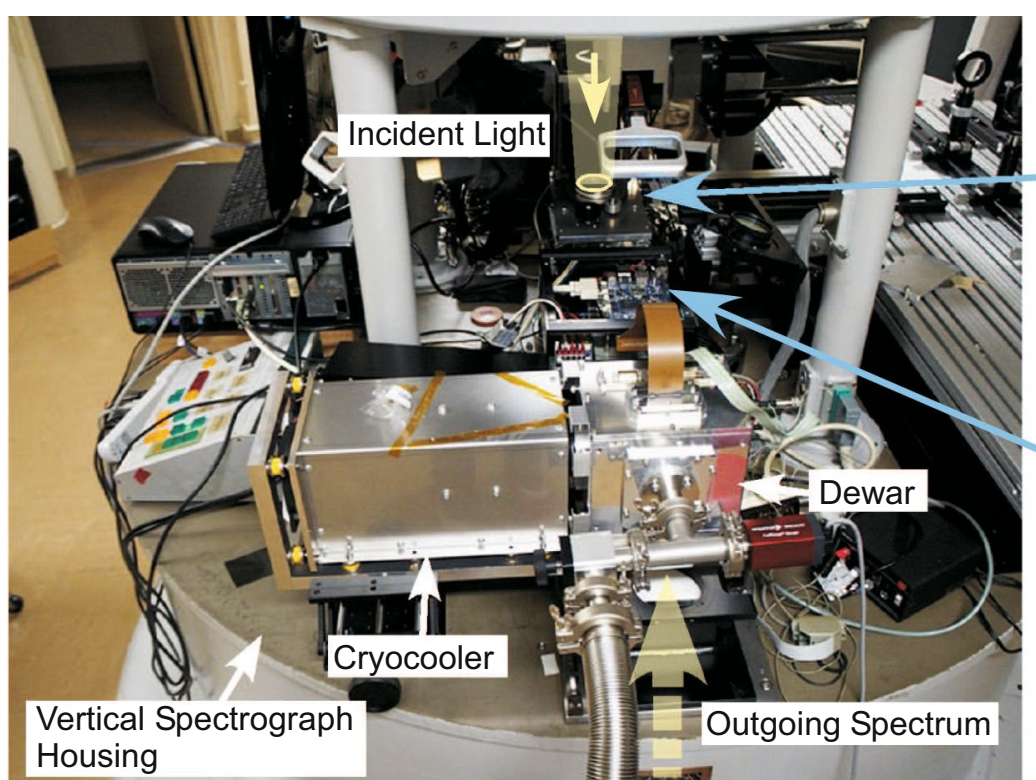

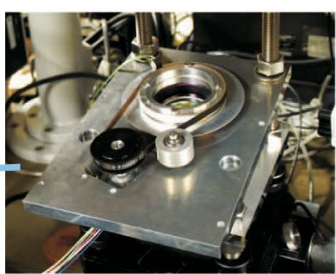

Rotating waveplate in front of the slit

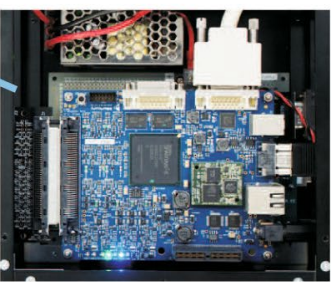

MACIE card and peripheral electronics

Fig. 4 Experimental arrangement of the H2RG camera system in the Domeless Solar Telescope. The instruments were deployed on the top-table of the vertical spectrograph. Close-ups of the rotating waveplate and the MACIE card are also shown

as $1.7 \mu \mathrm{m}$, the thermal noise was sufficiently low at an operation temperature of approximately $-110^{\circ} \mathrm{C}$ (Katsukawa et al. private communication), while not as cold as the $\mathrm{LN}_{2}$ temperature $\left(77 \mathrm{~K},-196^{\circ} \mathrm{C}\right)$. To prevent rapid temperature changes, which can result in a failure of the H2RG, the control input of the cryocooler was gradually increased and decreased by a microcomputer. In addition, aluminum blocks were attached to the back plate of the H2RG to increase the heat capacity of the H2RG unit to compensate for any problem due to the rapid change of the cryocooler temperature.

The DST is a vacuum solar telescope with a $60-\mathrm{cm}$ primary mirror. We installed the camera into the vertical spectrograph of the DST as shown in Fig. 4. The solar light from the telescope arrives from above at a slit located at the top of the spectrograph. The incident light travels downward into the spectrograph and the reflected outgoing spectrum exits upwardly. We positioned the dewar containing the H2RG at the exit focal plane of the spectrograph. The length of the spectrograph slit is $5 \mathrm{~cm}$, which corresponds to the field angle of $5^{\prime}$. Therefore, the H2RG, with an area of $37 \mathrm{~mm} \times 37 \mathrm{~mm}$, covers a field of view of $3^{\prime} .7$ along the slit with a spatial sampling of $0^{\prime \prime} .11$ pixel $^{-1}$. The dispersion is $0.039 \mathrm{~nm} \mathrm{~mm}^{-1}\left(0.7 \mathrm{pm}\right.$ pixel $\left.^{-1}\right)$ at $1083.0 \mathrm{~nm}$ (using the second order of the dispersion grating) and $0.1 \mathrm{~nm} \mathrm{~mm}^{-1}$ $\left(1.8 \mathrm{pm} \mathrm{pixel}^{-1}\right)$ at $1564.8 \mathrm{~nm}$ (first order). The polarization modulator is a rotating waveplate with $1 / 4-\lambda$ retardation at $1.6 \mu \mathrm{m}$ and with a retardation of approximately $1 / 3-\lambda$ at $1083.0 \mathrm{~nm}$. Although the waveplate could be rotated rapidly (Hanaoka 2012), the rotation rate of the waveplate was set as $1.8 \mathrm{rps}$ to accumulate a sufficient amount photoelectrons during the exposure. As 16 exposures were made during every rotation, the frame rate was $\sim 29 \mathrm{fps}$ as mentioned in the previous section. As an analyzer, a linear polarizer was installed in front of the slit behind the polarization modulator. This polarimeter had a single-beam configuration using only one of the orthogonal linear polarizations.

Using this arrangement, experimental studies with the polarimeter were successfully performed in the He I 1083.0-nm and Fe I 1564.8-nm wavelengths in 2018 and in 2019. These years were in the solar minimum phase, and there were no sunspots or conspicuous filaments in any of these years during our observational periods. Therefore, in this paper, we show a polarimetry result of a weak plage region (known as NOAA 12727) without sunspots using the wavelengths including Si I 1082.7-nm and He I 1083.0-nm lines. Figures 5a, b show an example of the Stokes $I$ spectra and a corresponding Stokes VII spectrum, respectively. The Si I 1082.7-nm line providing photospheric magnetic field information and the He I 1083.0-nm line providing chromospheric magnetic field information can be found in the spectra. A total of 480 images (16 exposures $\times 30$ rotations) were obtained at one slit position during $17 \mathrm{~s}$. The data were integrated into 8 images corresponding to a cycle of the polarization modulation, and they were converted to a set of images presenting Stokes signals. Even though the data were taken with insufficient light intensity, the noise 

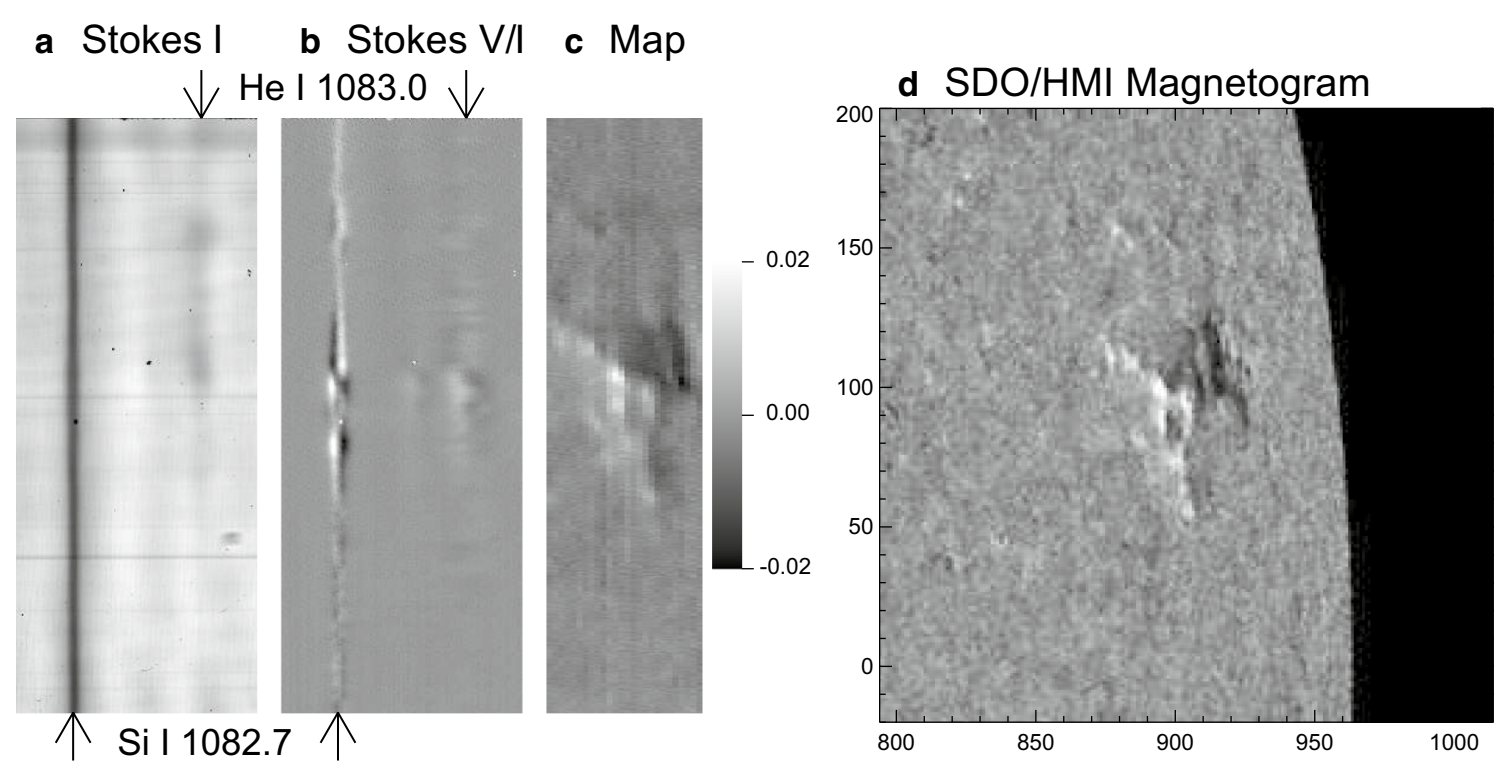

Fig. 5 Results of an experimental observation on 2018 November 20. a, b, respectively, show the Stokes / and V/I spectra of a wavelength range including the Si I $1082.7 \mathrm{~nm}$ and He I 1083.0-nm lines acquired at a slit position. c shows a Stokes V/I map of the blue wing of the Si I 1082.7-nm line composed from the data acquired at 26 slit positions, covering a plage region NOAA 12727 . The field of view is $3^{\prime} .7$ (along the slit) $\times 52^{\prime \prime}$. $\mathbf{d}$ shows a map of the longitudinal magnetic field acquired by SDO/HMI. The solar north is rotated counterclockwise from the top by $6^{\circ}$. A part of a HMI full-disk map including the field of view of (c) is shown

level of the Stokes signals is as low as $3 \times 10^{-3}$; because the signal-to-noise ratio is mainly limited by the photon statistics, a lower noise level is expected with a sufficient number of photons. In the Stokes V/I spectrum (Fig. 5b), remarkable Zeeman polarization signals in the Si I 1082.7-nm line and weak Zeeman signals both in the blue $(1082.91 \mathrm{~nm})$ and red $(1083.03 \mathrm{~nm})$ components of the He I 1083.0-nm line can be seen. We acquired such spectral data at 26 slit positions separated at every $2^{\prime \prime}$; as a result, we obtained polarization data of a two-dimensional field of view of $3^{\prime} .7 \times 52^{\prime \prime}$.

Figure 5c shows a Stokes V/I map of Si I $1082.7 \mathrm{~nm}$ of this field of view, which represents the photospheric longitudinal magnetic field of the weak plage region. The black and white patterns correspond to a bipolar magnetic polarity distribution (negative and positive polarities, respectively) in the plage region. Figure $5 \mathrm{~d}$ shows a longitudinal magnetogram obtained by the Helioseismic and Magnetic Imager (HMI; Scherrer et al. 2012) of the Solar Dynamics Observatory (SDO; Pesnell et al. 2012) including the same area. The HMI uses photospheric magnetic field data based on the Fe I 617.3-nm line. It is confirmed that results from the HMI magnetic field map and the Stokes V/I map recorded with the infrared camera are in good agreement.

The result of this experiment confirms the high polarimetric performance of the new infrared camera. Nevertheless, certain problems, such as the degradation of the image quality due to the noise from the cryocooler, have been revealed. This noise is a kind of electromagnetic interferences, which increases in proportion to the output of the cryocooler (the data shown in Fig. 5 were taken while the cryocooler was turned off). Now we are improving the grounding and the isolation to cope with this noise. After addressing these problems, the camera will be introduced to regular observations.

\section{Conclusions}

We successfully constructed a polarimeter system with a H2RG array by Teledyne, which is a large-format, highspeed, near-infrared detector suitable for solar polarimetry. Although it has been difficult to synchronize H2RGs under fast readout mode with external devices, such as a polarization modulator, we solved this problem by introducing a MACIE card and relevant assembly codes provided by Markury Scientific. With this development, advanced solar polarimetry is realized, which will enable the tracking of the evolution of the magnetic field in solar filaments, which occasionally erupt and develop into CMEs.

The synchronous operation of the SIDECAR/H2RG with an external device under fast readout mode can be realized using an existing SIDECAR/H2RG without preparing a new, dedicated camera system. Only the addition of a set of a MACIE card and assembly codes is required. 


\section{Therefore, the technology of this camera system can be widely applied in near-infrared solar polarimetry.}

\begin{abstract}
Abbreviations
H2RG: HAWAll-2RG; fps: Frames per second; MACIE: Markury ASIC control and interface electronics; TIP: Tenerife infrared polarimeter; CMEs: Coronal mass ejections; NAOJ: National astronomical observatory of Japan; A/D: Analogto-digital; SIDECAR: System for image digitization, enhancement, control and retrieval; SAM: SIDECAR acquisition module; ASIC: Application-specific integrated circuit; PC: Personal computer; CPLD: Complex programmable logic device; CDS: Correlated double sampling; DST: Domeless solar telescope; HMI: Helioseismic and Magnetic Imager; SDO: Solar dynamics observatory.

\section{Acknowledgements}

The camera system was developed and tested with the support of the Advanced Technology Center of the NAOJ. The authors thank Markus Loose from Markury Scientific for his help in successfully implementing the MACIE to the H2RG and the SIDECAR ASIC. The Hida Observatory of Kyoto University accepted our proposal for the experimental observations, and the observations were carried out with the support of the staff of Hida Observatory. We thank the SDO team for providing the HMI data; SDO is a mission for NASA's Living With a Star program. We would like to thank Editage (www.edita ge.com) for English language editing.
\end{abstract}

\section{Authors' contributions}

$\mathrm{YH}$ led the development of the infrared camera. YKat was the point of contact to Teledyne and Markury Scientific. MS worked on assembling and experiments, YKam contributed to establishing the cryogenic performance of the dewar, and NI performed experiments for the temperature control of the dewar. All authors read and approved the final manuscript.

\section{Funding}

This work is supported by a Japanese Kakenhi grant 15H05814,"Project for Solar-Terrestrial Environment Prediction" and also by a NAOJ research grant.

\section{Availability of data and materials}

The datasets generated during the current study are not publicly available because they are for experimental purpose; however they are available from the corresponding author on reasonable request.

\section{Competing interests}

The authors declare that they have no competing interests.

\section{Author details}

${ }^{1}$ National Astronomical Observatory of Japan, 2-21-1 Osawa, Mitaka, Japan.

${ }^{2}$ University of Tokyo, 7-3-1 Hongo, Bunkyo, Tokyo, Japan.

Received: 4 July 2020 Accepted: 19 November 2020

Published online: 26 November 2020

\section{References}

Blank R, Anglin S, Beletic JW, Bhargava S, Bradley R, Cabelli CA, Chen J, Cooper D, Demers R, Eads M, Farris M, Lavelle W, Luppino G, Moore E, Piquette E, Ricardo R, Xu M, Zandian M (2012) H2RG focal plane array and camera performance update. In: Holland AD Beletic JW (eds) Proc. SPIE High Energy, Optical, and Infrared Detectors for Astronomy V. 8453:845310-116. https://doi.org/10.1117/12.926752

Chen J, Loose M, Ricardo R, Beletic J, Farris M, Xu M, Wong A, Cabelli C (2014) SIDECAR ASIC firmware for astronomy applications. In: Proc. SPIE High Energy, Optical, and Infrared Detectors for Astronomy VI. 9154:915426-1. https://doi.org/10.1117/12.2056481

Collados M, Lagg A, Díaz García JJ, Hernández Suárez E, López López R, Páez Mañá E, Solanki SK (2007) Tenerife Infrared Polarimeter II. In: Heinzel P
Dorotovič I Rutten RJ (eds) The Physics of Chromospheric Plasmas, ASP Conference Series 368:611-616

Collados M, López R, Páez E, Hernández E, Reyes M, Calcines A, Ballesteros E, Díaz JJ, Denker C, Lagg A, Schlichenmaier R, Schmidt W, Solanki SK, Strassmeier KG, von der Lühe O, Volkmer R (2012) GRIS: The GREGOR Infrared Spectrograph. Astronomische Nachrichten 333:872-879. https:// doi.org/10.1002/asna.201211738

Hanaoka Y (2012) Polarimeter with a high-speed rotating waveplate for the solar observation. In: McLean IS Ramsay SK Takami H (eds) Proc. SPIE Ground-based and Airborne Instrumentation for Astronomy IV. 8446:844670-1-8. https://doi.org/10.1117/12.925304

Hanaoka Y, Katsukawa Y, Morita S, Kamata Y, Ishizuka N (2019) Development of an Infrared Camera Using a Hawaii-2RG Detector for Solar Polarimetry. In: Gandorfer A Lagg A Raab K (eds) Proceedings of the 9th Solar Polarization Workshop SPW9. https://doi.org/10.17617/2.3194859

Hanaoka Y, Sakurai T (2017) Statistical study of the magnetic field orientation in solar filaments. Astrophys J 851:130-1-11. https://doi.org/10.3847/15384357/aa9cf1

Harvey J, Hall D (1975) Magnetic field observations with Fe I 215648 Å. Bull Am Astro Soc 7:459

Jaeggli SA, Lin H, Mickey DL, Kuhn JR, Hegwer SL, Rimmele TR, Penn MJ (2010) FIRS: a new instrument for photospheric and chromospheric studies at the DST. Mem S A It. 81:763-768

Lagg A (2007) Recent advances in measuring chromospheric magnetic fields in the He I 10830 Åline. Adv Space Res 39:1734-1740. https://doi. org/10.1016/j.asr.2007.03.091

Lagg A, Lites B, Harvey J, Gosain S, Centeno R (2017) Measurements of Photospheric and Chromospheric Magnetic Fields. Space Sci Rev 210:37-76. https://doi.org/10.1007/s11214-015-0219-y

Loose M, Smith B, Alkire G, Joshi A, Kelly D, Siskind E, Mann S, Chen J, Askarov A, Fox-Rabinovitz J, Leong E, Goodwin A, Lindsay D, Rossetti D, Mah J, Cheng E, Miko L, Culver H, Wollack E, Content D (2018) The ACADIA ASIC: detector control and digitization for the Wide-Field Infrared Survey Telescope (WFIRST). In: Proc. SPIE Astronomical Telescopes + Instrumentation. 10709:107090T. https://doi.org/10.1117/12.2313067

Mártinez Pillet V, Collados M, Sánchez Almeida J, González V, Cruz-Lopez A, Manescau A, Joven E, Paez E, Diaz J, Feeney O, Sánchez V, Scharmer G, Soltau D (1999) LPSP \& TIP: Full Stokes Polarimeters for the Canary Islands Observatories. In: Rimmele TR, Balasubramaniam KS, Radick RR (eds) High Resolution Solar Physics: Theory, Observations, and Techniques, ASP Conference Series 183:264-272

Mosby, G, Eggen N, Wolf M, Jaehnig K, Kotulla R (2016) Persistence characterization and data calibration scheme for the RSS-NIR H2RG detector on SALT. In: Proc. SPIE 9915:99152U. https://doi.org/10.1117/12.2233227

Nakai Y, Hattori A (1984) Domeless Solar Tower Telescope at the Hida Observatory. Mem Fac Sci Kyoto Univ Series of Phys Astrophys Geophys Chem 36:385-399

Penn MJ (2014) Infrared solar physics. Liv Rev Solar Phys 11:2. https://doi. org/10.12942/lrsp-2014-2

Pesnell WD, Thompson BJ, Chamberlin PC (2012) The solar dynamics observatory (SDO). Solar Phys 275:3-15. https://doi.org/10.1007/s1120 7-011-9841-3

Sakurai T, Hanaoka Y, Arai T, Hagino M, Kawate T, Kitagawa N, Kobiki T, Miyashita M, Morita S, Otsuji K, Shinoda K, Suzuki I, Yaji K, Yamasaki T, Fukuda T, Noguchi M, Takeyama N, Kanai Y, Yamamuro T (2018) Infrared spectropolarimeter on the Solar Flare Telescope at NAOJ/Mitaka. Publ Astron Soc Japan 70:58-1-58-17. https://doi.org/10.1093/pasj/psy050

Scherrer PH, Schou J, Bush RI, Kosovichev AG, Bogart RS, Hoeksema JT, Liu Y, Duvall TL, Zhao J, Title AM, Schrijver CJ, Tarbell TD, Tomczyk S (2012) The helioseismic and magnetic imager (HMI) investigation for the solar dynamics observatory (SDO). Solar Phys 275:207-227. https://doi. org/10.1007/s11207-011-9834-2

\section{Publisher's Note}

Springer Nature remains neutral with regard to jurisdictional claims in published maps and institutional affiliations. 